\title{
Rapid development of recurrences after partial nephrectomy for papillary renal cell carcinoma initially presented as Wunderlich syndrome
}

\author{
Rehan T Gamage ${ }^{1}$, Jayani Malagala ${ }^{2}$, Dhammika Ranasingha ${ }^{2}$, Anuruddha M Abeygunasekera ${ }^{2}$ \\ ${ }^{1}$ National Hospital of Sri Lanka, Colombo. \\ ${ }^{2}$ Colombo South Teaching Hospital, Sri Lanka
}

Keywords: : peri-renal haematoma; renal tumour; papillary renal cell carcinoma; Wunderlich syndrome

\section{Introduction}

Renal cell carcinoma (RCC) represents $2-3 \%$ of all malignancies. It is the commonest solid lesion of the kidney and accounts for $90 \%$ of all kidney malignancies. Even though RCC classically presents as a triad of flank pain, haematuria and palpable mass, with the availability and common use of imaging techniques, more than $50 \%$ are diagnosed incidentally. Nephron sparing surgery in the form of partial nephrectomy is recommended for early renal tumours to avoid long term renal insufficiency. Rarely RCC presents as a spontaneous renal haematoma (SRH) in subcapsular or peri-renal spaces which are known as Wunderlich syndrome [1]. In such situations whether nephron-sparing surgery is an oncologically adequate option is unknown.

\section{Case presentation}

A 40-year old man with sudden onset left loin pain was admitted to the emergency department. Severe, continuous and non-radiating pain was associated with vomiting and dizziness. There was no history of even trivial trauma. The patient was not known to have medical illnesses including diabetes mellitus, hypertension or hypercholesterolemia and family history was unremarkable. He was a cigarette smoker with 20 pack years. His BMI was $29.6 \mathrm{kgm}-2$. Pulse rate was $88 \mathrm{bpm}$ and BP was $150 / 90 \mathrm{mmHg}$. Abdominal examination was normal except for left renal angle tenderness.

Haemoglobin level was $11.3 \mathrm{~g} / \mathrm{dl}$ and serum creatinine were $95.3 \mu \mathrm{mol} / 1$. Abdominal ultrasonography revealed a mass in the left kidney with a peri-renal haematoma. A CT urogram confirmed the peri-renal haematoma. There was an underlying heterogeneous, exophytic mass measuring $4.8 \mathrm{~cm}$ with little contrast enhancement in the lower pole of the left kidney (Figure 1a). There was no fat in the mass to suggest an angiomyolipoma.

Correspondence: Rehan T Gamage

E-mail: r8rehan@gmail.com

(D)https://orcid.org/0000-0002-0649-1336

Received: 20-04-2020 Accepted: 15-06-2020

DOI: http://doi.org/10.4038/sljs.v38i2.8684

(c) (i)

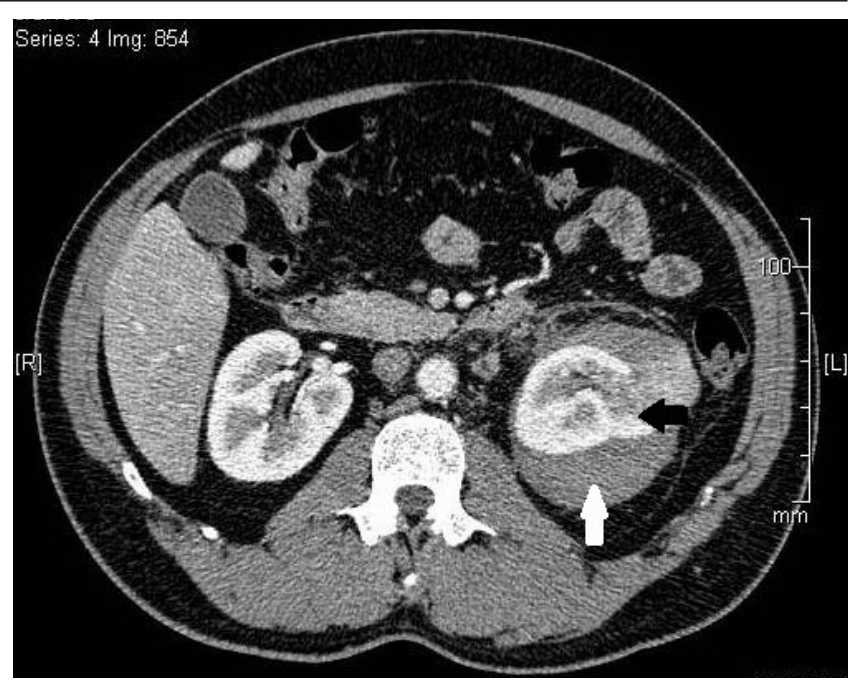

Figure 1a. CT Urogram showing left renal mass (black arrow) and peri-nephric haematoma (white arrow)

Figure 2a. CT urogram showing the tumour recurrence in the remaining kidney (arrow)

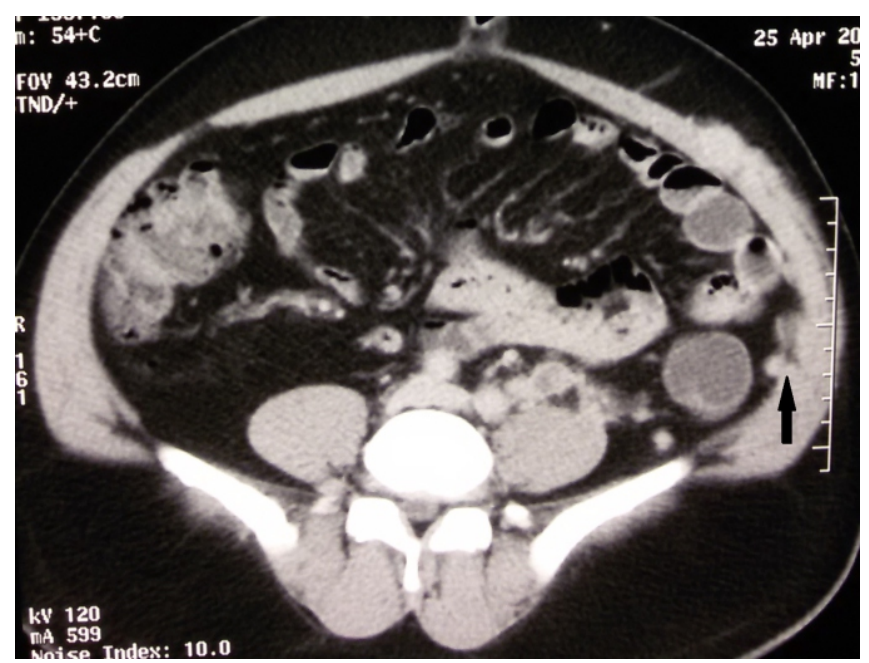

Figure 1b. Histology of the tumour showing papillary type II renal cell carcinoma. (H\&E x200)

Figure 2b. CT Urogram showing the recurrent tumour in the abdominal wall (arrow)

He was managed with analgesics, bed rest, intravenous fluids and closely monitored for early detection of haemodynamic instability. Size of the haematoma was assessed with regular ultrasound scans but was not progressing. As the CT scan was suspicious of malignancy and the RENAL nephrometry score 
was 6, partial nephrectomy was planned. During the surgery tissue planes were not clear and the organized blood clot was evacuated exposing a solid exophytic mass of the underlying kidney. As planned, partial nephrectomy was performed.

Histopathological assessment of the tumour revealed a papillary type II renal cell carcinoma with a diameter of 4.5 $\mathrm{cm}$ extending up to the renal capsule without infiltration (Fuhrman grade 2, T1b stage) (Figure 1b). The closest resection margin was $4 \mathrm{~mm}$ from the tumour.

Seven months later the patient noticed a lump deep to the surgical scar. A CT Urogram showed a recurrent tumour in the remaining kidney as well as multiple metastatic deposits in the liver and subcutaneous tissues beneath the surgical scar (Figures 2a \& 2b). He underwent completion nephrectomy and excision of subcutaneous nodules. The histopathology confirmed those to be recurrences of a papillary type II renal cell carcinoma. Thereafter he was treated with pazopanib orally. Eight months later he died of widespread metastases.

\section{Discussion}

Spontaneous renal haematoma (SRH) was originally described by Bonet in 1700 and further explored by Wunderlich in 1856. It is a rare condition in which there is haemorrhage into the subscapular or peri-renal space in the absence of trauma [1,2]. Acute flank pain, internal bleeding and abdominal tenderness are described as the classic presentation of SRH (Lenk's triad). Nevertheless, abdominal pain, haematuria and hypovolaemic shock were observed more commonly [2].

According to a meta-analysis involving 165 patients with spontaneous peri-renal haemorrhage, $61 \%$ of SRH were caused by neoplastic lesions while the rest were vascular, infections and idiopathic in origin [3]. Benign and malignant neoplasms contributed equally, commonest being angiomyolipoma and $\mathrm{RCC}$ respectively. However, the prevalence of spontaneous renal haematoma as a complication of tumours low. It is around $1 \%$ in renal cell carcinoma and much higher in angiomyolipoma [3]. The extensive necrosis regularly found in papillary renal cell carcinoma can cause rupture of the tumour followed by haemorrhage [4]. It could be spontaneous or follow minimal trauma.

The rarity and nonspecific presentation of Wunderlich syndrome possess a challenge when arriving at a diagnosis. CT urogram is considered the gold standard for establishing the diagnosis, and often useful to elucidate the underlying aetiology. The main criterion for diagnosis is bleeding in the sub-capsular and peri-renal space [5]. However current studies have shown that the diagnostic accuracy of RCC by $\mathrm{CT}$ is reduced to $60 \%$ when presenting with haemorrhage which is above $90 \%$ otherwise [5].

In general, papillary renal carcinoma has a better prognosis than clear cell variety and has a five-year cancer-specific survival rate of $80.5 \%$ though type II variety as in this patient has a poorer outcome than type I [6]. Despite that, the patient developed local recurrences and metastases within seven months. Therefore, in cases of peri-renal haematoma due to possible tumour rupture at the time of presentation, partial nephrectomy appears to be inadequate with the potential for tumour dissemination and seedling. Radical nephrectomy could be considered a more appropriate therapeutic option which may reduce the chances of local recurrences in such cases. In these cases, though there is no microscopic evidence of tumour infiltrating the renal capsule and invading the perirenal fat, staging the tumour as T1 may be an underestimation.

\section{Conclusion}

In conclusion, though renal cell carcinoma can be diagnosed by CT urogram preoperatively with high accuracy, the sensitivity of the test falls to around $60 \%$, in the presence of a peri-renal haematoma. Hence in cases of spontaneous perirenal haematoma, it is important to entertain the possibility of a malignant cause to avoid undue delay in treatment. In cases of peri-renal haematoma due to underlying malignancy, partial nephrectomy appears to be inadequate as it may predispose to tumour dissemination and local seedling.

All authors disclose no conflict of interest. The study was conducted in accordance with the ethical standards of the relevant institutional or national ethics committee and the Helsinki Declaration of 1975, as revised in 2000 .

\section{References}

1. Ebner, Suebert. Wunderlich CR. Handbuch der Pathologie und Therapie. 2nd ed.1856.

2. Albi G, Del Campo L, Tagarro D. Wunderlich's syndrome: causes, diagnosis and radiological management. Clin Radiol 2002;57:840-845. https://doi.org/10.1053/crad.2002.0981

3. Zhang JQ, Fielding JR, Zou KH. Etiology of spontaneous perirenal hemorrhage: a meta-analysis. J Urol 2002;167:15931596. https://doi.org/10.1016/S0022-5347(05)65160-9

4. Hora M, Hes O, Klecka J, Boudová L, et al. Rupture of papillary renal cell carcinoma. Scand J Urol Nephrol 2004; 38:481-484. https://doi.org/10.1080/00365590410018648

5. Sebastia MC, Perez-Molina MO, Alvarez-Castells A, Quiroga S, Pallisa E. CT evaluation of underlying cause in spontaneous subcapsular and perirenal hemorrhage. Eur Radiol 1997;7:686690 https://doi.org/10.1007/BF02742926

6. Steffens Sandra, Janssen Martin, Ross Fredrik C, et al. Incidence and long-term prognosis of papillary compared to clear cell renal cell carcinoma - A multicentre study. Eur J Cancer 2012; 48: 2347-52. https://doi.org/10.1016/j.ejca.2012.05.002 
Learning Points:

- Underlying malignant pathology should be considered in a spontaneous renal haematoma.

- Radical surgery is recommended for RCC presented with peri-renal haematoma. 